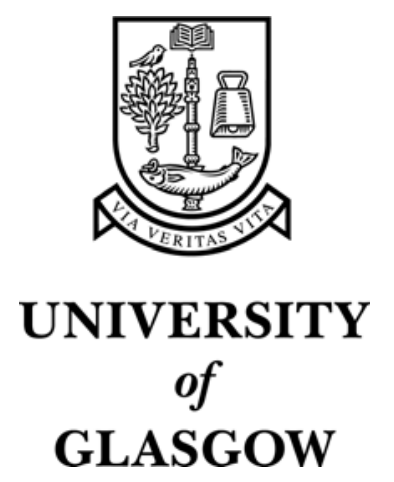

Johnson, N.P. and Khokhar, A.Z. and Chong, H.M. and Jin, C. and Mandel, J. and McMeekin, S. and De La Rue, R.M. (2005) Increasing optical metamaterials functionality. In, Szoplik, T., Eds. Metamaterials, 30-31 August 2005. Proceedings of SPIE--the International Society for Optical Engineering Vol 5955, pages pp. 163-168, Warsaw, Poland.

http://eprints.gla.ac.uk/3702/ 


\title{
Increasing Optical Metamaterials Functionality
}

\author{
Nigel P Johnson, Ali Z Khokhar, Harold M Chong, Chongjun Jin, Jharna Mandel, \\ Scott McMeekin* and R M De La Rue \\ Department of Electronics \& Electrical Engineering, University of Glasgow, \\ Glasgow, G12 8LT, UK. Email njohnson@elec.gla.ac.uk \\ *School of Engineering Science and Design Glasgow Caledonian University, \\ Glasgow G4 0BA, UK
}

\begin{abstract}
Gold Split Ring Resonators (SRRs) were fabricated on silicon substrates by electron beam lithography and liftoff, with overall dimensions of approximately $200 \mathrm{~nm}$. Reflectance spectra from the SRRs are similar to those published elsewhere. New devices are proposed based on the additional functionality afforded by the use of a silicon substrate.
\end{abstract}

Key words Split Ring Resonators, Silicon, Gold

\section{INTRODUCTION}

Left-handed materials, as first theoretically proposed by Veselago ${ }^{1}$ or 'metamaterials', in the form of arrays of Split Ring Resonators (SRRs) and wires as proposed by Pendry ${ }^{2}$, have been shown to have negative indices of refraction arising from negative electrical permittivity $\varepsilon$, and negative magnetic permeability $\mu$. Electromagnetic (EM) radiation encountering these metamaterials follows Snell's law except that the index of refraction is negative and consequently bends EM radiation in the opposite sense to conventional right-handed materials. Such metamaterials were initially realised in the microwave region by $\mathrm{Smith}^{3}$, but efforts have recently been directed towards the infra-red and visible region of the spectrum ${ }^{4}$. Negative refraction has also been demonstrated by photonic crystals $(\mathrm{PhC})$, but this is via a different mechanism where by the dispersion of the band structure of the PhC is used to achieve negative refraction ${ }^{5,6}$.

Focusing effects have also been demonstrated, with light from the visible being used to image subwavelength features through a slab of silver ${ }^{7}$, making use of the negative $\varepsilon$ properties of the metal. It is sufficient in this case for just the permittivity to be negative because, in the near field, the electric and magnetic responses of materials are decoupled. If a material with negative $\mu$ and negative $\varepsilon$ can be fabricated in the visible range then the realisation of a perfect lens could be possible, in which the image is not limited by diffraction because the near field information is preserved. This behaviour in itself may prove useful in nano fabrication procedures, however the question posed here is: what functionality can we envisage beyond simple imaging? In order to contemplate more functionality we have changed the substrate from glass to one offering more possibilities, namely silicon.

Firstly we consider the problems of fabricating sufficiently small SRRs to operate in the visible range. SRRs have been fabricated by e-beam lithography and lift-off which show electric and magnetic resonances at 1.5 and $3 \mu \mathrm{m}$-these are from structures around $320 \mathrm{~nm}$ in length and $70 \mathrm{~nm}$ wire width ${ }^{4}$. 
While shrinking the devices is still possible it becomes more difficult to shift the frequency of the resonances into the visible. Because the gap in the ring becomes smaller the capacitance increases and subsequently the frequency decreases. However it has recently been shown ${ }^{8}$ that as the gap is opened in the individual rings of the SRRs the capacitance is reduced and the resonance frequency is increased and more so when the structure is formed by just two parallel cut wires with the same dimension of the length of the rings. This transmutation has the disadvantage of reducing the ratio of wavelength to lattice constant from 10 to 2 and moves further away from the metamaterial regime. The capacitance of the structure maybe expected to increase if a higher dielectric constant material is used as a substrate.

Linden et $\mathrm{al}^{4}$ have reported the excitation of the LC resonance, in a SRR with normal-incidence geometry, by coupling of the electric field of the light to the capacitors. Other simulations for an inplane parallel geometry show a frequency range for negative magnetic permeability $\mu$ when the magnetic field couples to the LC resonance. Together with an electric response that has negative permittivity $\varepsilon$, it becomes possible to obtain materials with negative index of refraction. Though ultimately the geometry where incident light is perpendicular to the plane of the SRRs geometry used here is an insufficient condition for exploitation of negative index.

\section{EXPERIMENTAL}

We began our investigation with the fabrication of smaller size-scale sets of SRRs. Linden et al identified two types of polarisation-dependent resonances when the SRRs were illuminated normal to the plane. The first reflectance peak around $1.4 \mu \mathrm{m}$ displays a resonance that broadens and shifts to longer wavelengths when the electric field is changed between perpendicular to the SRR gap and parallel to the gap ${ }^{4}$. The SRRs in figure 1 (a) at $1.3 \mu \mathrm{m}$ show similar polarisation dependence. These resonances (reflection peaks) are associated with the plasmon resonance of a thin finite wire and are not associated with negative $\mu$. The resonance is stronger when the electric field is aligned along the length of two parallel sides of the rings rather than one side plus the wire with the gap. The second reflectance peak around $3.2 \mu \mathrm{m}$ disappears when TE is parallel to the gap.

The gaps in the measured spectra are due to limitations in the ranges of our two measuring systems with the range 1.6 to $2.5 \mu \mathrm{m}$ currently inaccessible. Although the structures shown in figure 1 are physically smaller than those reported in reference 4 , the important resonance at $3.2 \mu \mathrm{m}$ is only marginally shorter in wavelength. This due to the combination of the narrower gap in the rings giving larger capacitance and, possibly, the higher dielectric constant of the silicon substrate material. The thickness of the metal also has a small effect, with thinner material giving shorter wavelengths resonance. We note that recently smaller rings with a magnetic resonance around $1.5 \mu \mathrm{m}$ have been fabricated ${ }^{9}$. 
FDTD simulation results (RSoft Fullwave) are shown immediately below the respective measurements. The Drude model with a $5 \mathrm{~nm}$ grid and $1 \mu \mathrm{m}$ excitation pulse was applied (note change in wavelength scale for simulations). The results show a correlation between the experimental and simulated results however the simulations are up to $40 \%$ lower in wavelength than the measured values. For completeness the closed rings were also fabricated and measured. As expected there is no polarisation dependence and the spectra for both polarisations resemble those of the TE field parallel to the gap.

a

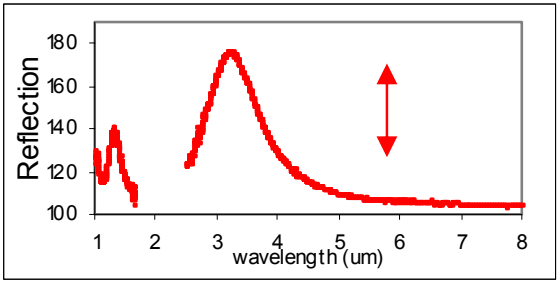

$\mathrm{b}$

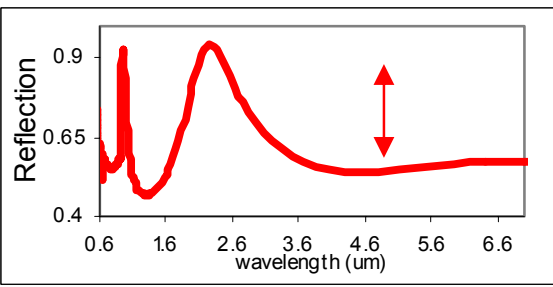

$\mathrm{C}$

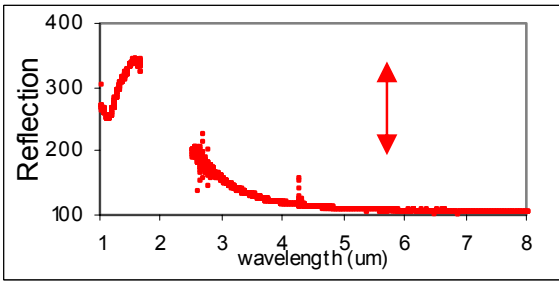

$\mathrm{d}$

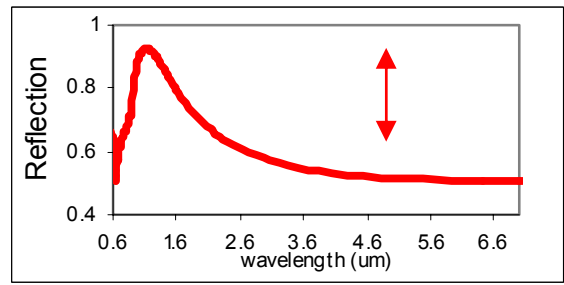

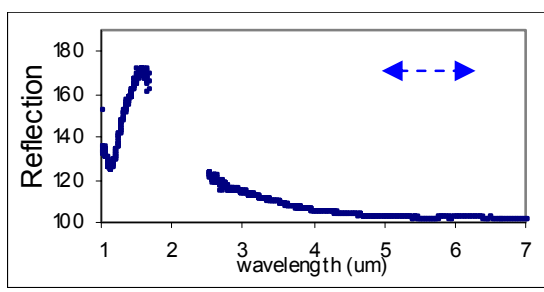
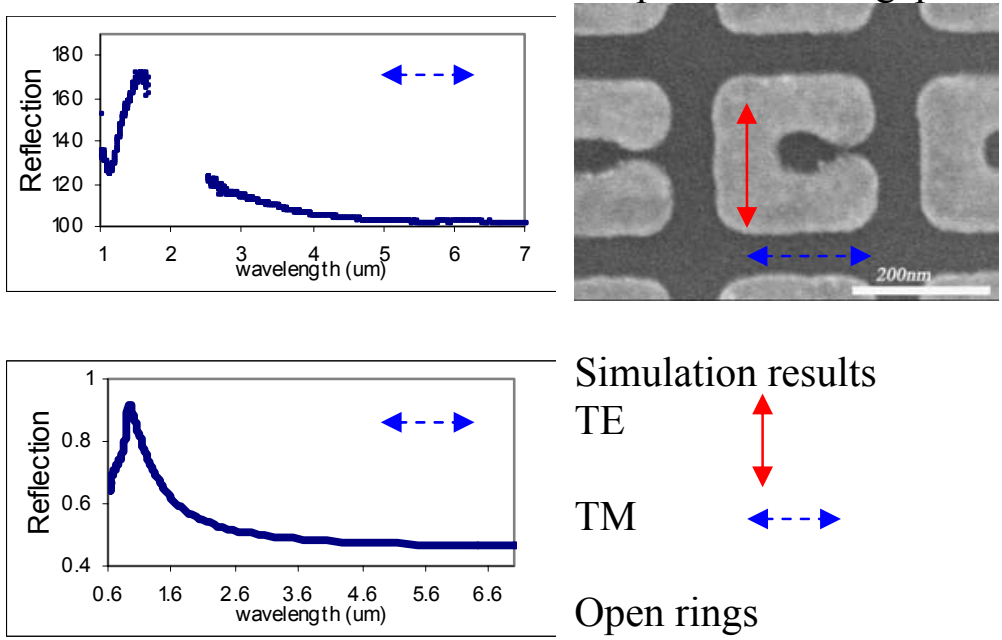

Simulation results

TE

TM

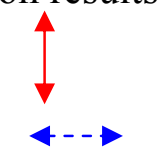

Open rings


Simulation results

TE

TM

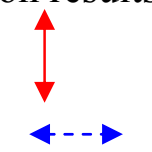

Closed rings

Figure 1 Set of $25 \mathrm{~nm}$ thick gold SRRs on silicon. Dimensions $l=217, a=297, w=66, d=33 \mathrm{~nm}$. Row (a) experimental reflection measurements for TE polarised light across the SRR gap; TE polarised parallel to the gap; SEM picture. Row (b), simulations as above. Row (c), experimental reflection measurements for closed rings both polarisations and SEM picture. Row (d), simulations for closed rings. ( $($ is the SRR length, a is the SRR period, $w$ is the wire width and $d$ is the gap). 


\section{ADDING FUNCTIONALITY}

Despite the possible frequency disadvantage of using silicon as a substrate it also has the potential advantage of adding functionality to the metamaterial. The electrically induced magnetic resonance is turned off when the ring is closed. If this ring could be opened and closed then the material properties could be controlled. Two possibilities are electrical and optical closure of the rings. Electrically controlled devices may be possible with MOSFET structures in which a conducting channel is created across the gap. However, multiple electrical connections to each SRR make this a complex route.
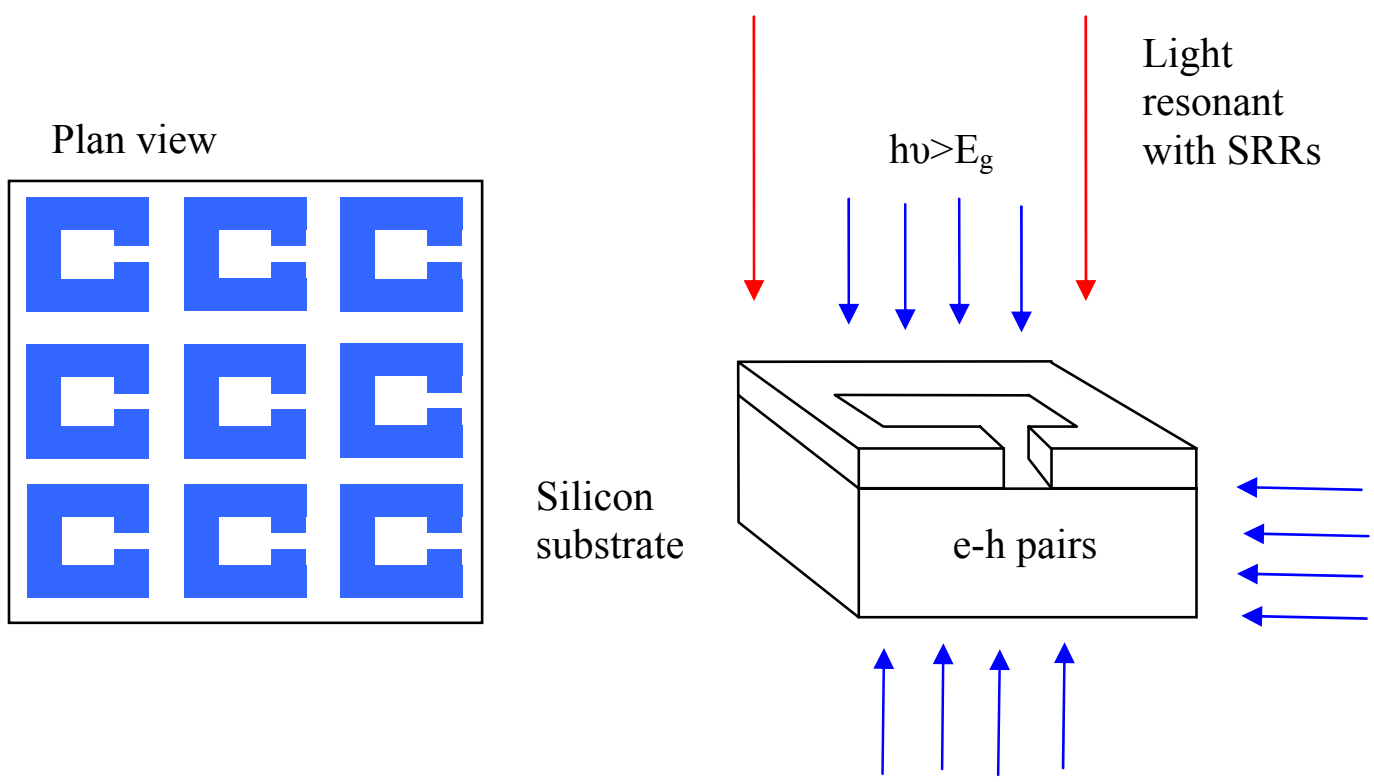

Figure 2 Schematic of operation of the device. Illumination with higher energy light than the electrical band gap of silicon $\mathrm{E}_{\mathrm{g}}$ is used to induce electron hole pairs providing a conduction path to optoelectronically close the rings. The refection and transmission of light at lower frequency, resonant with the SRRs, would be modulated by the higher frequency light

For rings constructed on silicon there is the possibility of inducing carriers in the material by additional light illumination above the band gap of silicon. This could be from above the substrate or below - or possibly guided from the side. For reflection of light at resonance the light could be modulated by the shorter wavelength light effectively - making an array of light transistors or switches. 
The schematic in figure 2 shows possible arrangements. While this approach would make it possible to be able to turn off the whole behaviour of the array of SRRs, addressing a single ring is much more challenging. One possibility is to guide light to individual rings (or sets of rings). Two-photon absorption could be exploited to allow individual addressing of sets of rings, since they are likely to be smaller that the guided regions with only sufficient power density for two photon absorption and carrier generation at the intersection of two illuminated waveguides shown schematically in figure 3 .

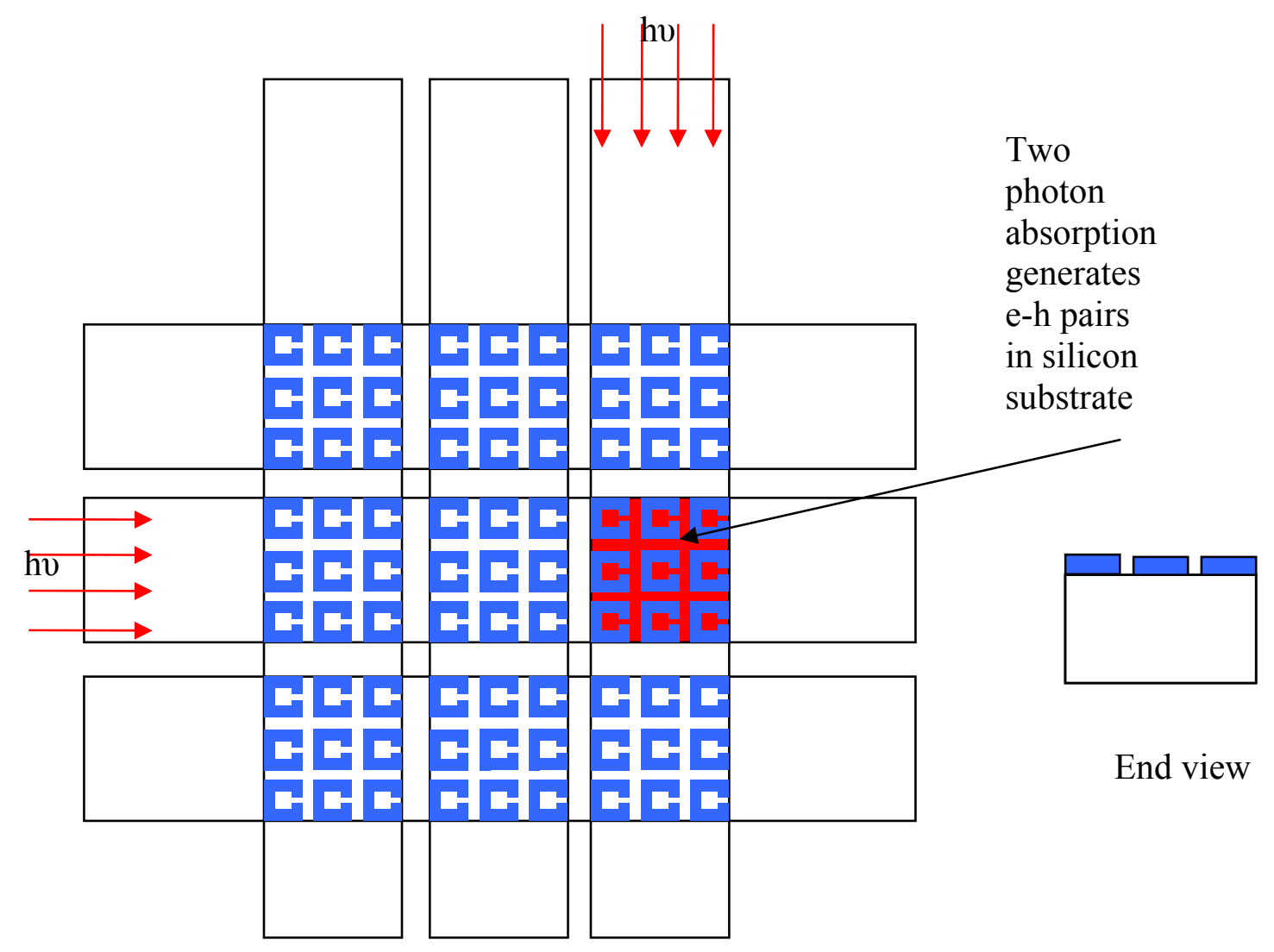

Figure 3 Schematic of addressable sets of SRRs light is guided from orthogonal directions to sets of SRRs. Only when there is illumination from both directions simultaneously are there sufficient photons for two-photon absorption and the creation of electron-hole pairs thereby switching one addressable set of SRRs. 


\section{CONCLUSIONS}

We have replicated the main results shown in reference 4 but on a different material system, silicon rather than silica. The use of silicon as a substrate could make it possible to increase the functionality of SRRs by switching them, together or individually. We give outline proposals of two schemes.

\section{Acknowledgements}

This work is supported by EU- Metamorphose NoE and simulations have been carried out with the support of RSoft.

\section{References}

\footnotetext{
${ }^{1}$ Veselago V G 1967 Usp. Fiz Nauk 92517. Veselago V G 1968 Sov. Phys - Usp 10 509. (English Translation)

${ }^{2}$ J. B. Pendry Phys. Rev. Lett., 85, 3966 (2000).

${ }^{3}$ D. R. Smith, Willie J. Padilla, D. C. Vier, S. C. Nemat-Nasser and S. Schultz, Phys. Rev. Lett., 84, 4184

${ }^{4}$ S Linden, C Enkrich, M Wegener,Ji Zhou, Thomas K, C M. Soukoulis Science 306, 1351 (2004).

${ }^{5}$ M. Notomi, Optical and Quantum Elec., 34, 133 (2002).

${ }^{6}$ P. Markos and C. M. Soukoulis Physica Status Solidi A, 197, 595 (2003).

${ }^{7}$ Nicholas Fang, Hyesog Lee, Cheng Sun, and Xiang Zhang Science 22 534-537 (2005).

${ }^{8}$ G Dolling, C Enkrich M Wegener, J Zhou, C M Soukoulis and S Linden Submitted to optics letters, Arxiv preprint physics/0507045, 2005.

${ }^{9}$ C. Enkrich, M. Wegener, S. Linden, S. Burger, L. Zschiedrich, F. Schmidt, J. Zhou, Th. Koschny, C.M. Soukoulis, Submitted to Phys. Rev. Lett.
} 